\title{
Strain Elastography - How To Do It?
}

\section{(ㄷ) (i) (으 $\Theta$}

Authors

Christoph F. Dietrich¹, Richard G. Barr², André Farrokh³, Manjiri Dighe4, Michael Hocke ${ }^{5}$, Christian Jenssen ${ }^{6}, Y_{i}$ Dong7, Adrian Saftoiu ${ }^{8}$, Roald Flesland Havre ${ }^{9}$

Affiliations

1 Caritas-Krankenhaus, Innere Medizin 2, Bad Mergentheim, Germany

2 Radiology, Northeastern Ohio Medical University, Rootstown, United States

3 Department of Breast Imaging and Interventions, University Hospital Schleswig-Holstein Campus Kiel, Kiel, Germany

4 Department of Biology, University of Washington, Radiology, Seattle, United States

5 HELIOS Klinikum Meiningen, Internal Medicine II, Meiningen, Germany

6 Krankenhaus Märkisch Oderland Strausberg/ Wriezen, Klinik für Innere Medizin, Wriezen, Germany

7 Zhongshan Hospital, Ultrasound, Shanghai, China

8 University of Medicine and Pharmacy of Craiova, Research Center of Gastroenterology and Hepatology, Craiova, Romania

9 Department of Medicine, Haukeland University Hospital, Bergen, Norway

Key words

ultrasound, endoscopic ultrasound, real-time, tissue elastography (TE)

received 28.05.2017

revised 27.08.2017

accepted 29.08.2017

\section{Bibliography}

DOI https://doi.org/10.1055/s-0043-119412

Ultrasound Int Open 2017; 3: E137-E149

(c) Georg Thieme Verlag KG Stuttgart · New York

ISSN 2199-7152

\author{
Correspondence \\ Dr. Christoph F. Dietrich, MD \\ Caritas-Krankenhaus, Innere Medizin 2 \\ Uhlandstr. 7 \\ 97980 Bad Mergentheim \\ Germany \\ Tel.: + 49/7931/58 2201, Fax: +49/7931/58 2290 \\ Christoph.Dietrich@ckbm.de
}

\section{ABSTRACT}

Tissue stiffness assessed by palpation for diagnosing pathology has been used for thousands of years. Ultrasound elastography has been developed more recently to display similar information on tissue stiffness as an image. There are two main types of ultrasound elastography, strain and shear wave. Strain elastography is a qualitative technique and provides information on the relative stiffness between one tissue and another. Shear wave elastography is a quantitative method and provides an estimated value of the tissue stiffness that can be expressed in either the shear wave speed through the tissues in meters/ second, or converted to the Young's modulus making some assumptions and expressed in $\mathrm{kPa}$. Each technique has its advantages and disadvantages and they are often complimentary to each other in clinical practice. This article reviews the principles, technique, and interpretation of strain elastography in various organs. It describes how to optimize technique, while pitfalls and artifacts are also discussed.

\section{Introduction to Elastography}

Even from the early stages of neoplastic development, cancerous tissue becomes stiffer as the geometry of the tissue matrix changes, desmoplastic reaction occurs with increased production of connective tissue, the density of blood vessels changes, and the cell density and intercellular junctions are also altered. Manual palpation can detect the large difference in stiffness found between cancerous lesions and normal tissues, especially in superficial areas such as the breast and the prostate, and has been considered an important part of clinical examination for hundreds of years. More recently, ultrasound elastography techniques have been developed that can image tissue stiffness, offering greater sensitivity for deeper structures and better spatial resolution than manual palpation and so have the potential for early-stage differentiation of benign and malignant tissue [1]. 


\section{What Elastography Techniques are Used?}

The methods currently used in commercially available equipment can be classified into two main types: strain imaging and shear wave speed measurement/imaging [2,3]. The different types of elastography vary both in the method used to exert the force (stress) and in the measurement of the subsequent tissue displacement (strain), but may be complementary because of their different physical properties, artifacts, limitations and the clinical applications for which they are best suited. The use of shear wave elastography is often preferred for the assessment of liver fibrosis [2-9].

\section{Technical Principles of Tissue Elastography}

Strain images are generated by using the transducer to apply repetitive minimal pressure to the tissues. The subsequent tissue displacement is tracked between pairs of RF echo frames and the strain calculated from the axial gradient of the displacements. Under an equal amount of stress, a stiff region experiences less strain (deformation) than surrounding softer tissue. Using a color map to code different magnitudes of strain, a two-dimensional strain image can be translucently superimposed on the conventional B-mode image, aiding the assessment of the spatial relationship between the ultrasound image and the elastography data. Quantitative elasticity measurements expressed in kPa cannot be obtained, as the local degree of stress is unknown. Thus, strain elastography is a qualitative technique in which relative stiffness differences in insonified tissue are displayed.

\section{Strain-Based Elastography - How Does it Work?}

Strain elastograms are generated by palpation with the imaging transducer or with physiological patient motion (breathing and heartbeat). It provides a natural extension of the B-mode imaging examination as both images can be displayed simultaneously. The most useful frames for interpreting strain images with good signalto-noise ratio are those with a constant rate of displacement, that is, during the time of downward or upward movement of the trans- ducer. The quality will be poorest, and sometimes completely absent, at the times when the compressional force is released to allow the tissue to return to its original shape due to the visco-elastic properties of soft tissue (see section: strain graph display).

Because biological tissue deforms in a non-linear manner, the soft tissues will appear stiffer as more pressure is used. Therefore, pre-compression should be minimal, with the transducer held lightly just in contact with the skin and plenty of gel applied [10]. Small amplitude movements are all that is required to induce the required strain of $0.1-2 \%$ in the tissue, and different manufacturers of strainbased imaging modalities may have optimized the strain mode for different strain levels within this interval. Most strain elastography platforms come with a display that provides real-time feedback about whether the appropriate amount of stress is being applied. Displacement that is both too large and too small will lead to poor elastograms. Also, sufficient stress must be provided to create sufficient strain at the region of interest.

An alternative to transducer palpation is to hold the transducer still and allow the internal physiological pulsations from cardiac, respiratory or muscle contractions to generate the strain. However, since displacements are measured primarily in an axial direction, better results are generally obtained when simple uniaxial stress is applied. Footplate extenders can improve the uniformity of the applied stress and maximize the depth of stress penetration [11] - Fig. 1.

\section{Definition of Color Coding, Why?}

Grayscale, single color and rainbow color maps among others are available to display the magnitude of strain in the image. Some users prefer to denote red as stiff (representing danger or alarm) and blue as soft, whereas others have chosen the reverse (blue allows better transparency to correlate the stiff region with the morphology seen in the B-mode image), so careful investigation of the labels used for the displayed color bar is necessary for correct interpretation of the elastogram. If a grayscale map is used, it should not be displayed over the grayscale B-mode image, but rather as a separate strain image or "elastogram".
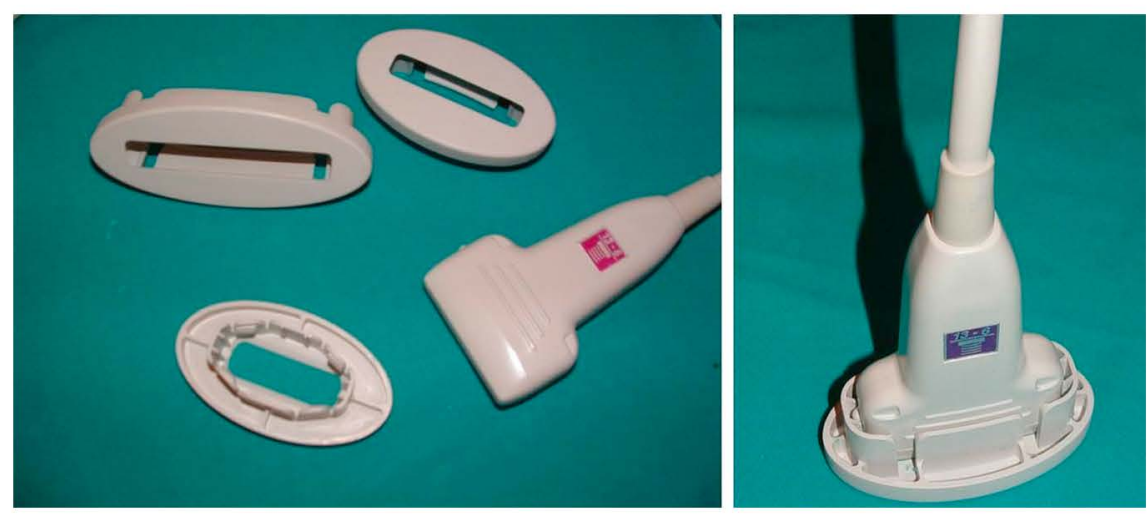

Fig. 1 Footplate extenders for linear probe 


\section{"Knobology"}

Knobology is not uniform among different manufacturers, and the level of strain image parameters available to the user may vary. Some systems only provide a scale of different strain levels which mainly adjusts depth, strain color representation and temporal overlap. However, some general principles for adjustable settings specific to strain imaging will apply across all systems.

\section{Frequency selection}

As with B-mode imaging, selecting a higher frequency transducer offers higher strain image resolution, but a lower frequency will offer better stress penetration for imaging lesions at a greater depth.

\section{Frame rate, palpation speed and amplitude}

The strain image quality can be optimized by adjusting the frame rate to match the palpation speed and amplitude in order to achieve the desired tissue strain contrast in the image. Most vendors have a numerical scale or bar to aid in finding the optimal palpation speed and amplitude.

\section{Noise filters and persistence}

Noise filters are used to reject poor quality pixels within each frame or the entire frame. The threshold for rejection may be under the control of the operator in which case increasing the threshold will result in less noisy elastograms but may contain more 'black holes' (rejected pixels in the frame) or flickering of the image (due to whole frame rejection). The persistence control can be used to create a more stable color display.

\section{Description of quality parameters}

\section{Strain graph display and 'press indicator'}

Manufacturers offer different methods including: a strain graph display, a numerical value, a bar, or a scale marking the optimal stress, with the aim of providing real-time feedback to the user on the degree and uniformity of the compression technique. Each system has a sweet spot regarding the amount of displacement and frequency of displacement to generate optimal elastograms.

\section{Adjustment of console controls}

- Ref Freq - Up, down toggle adjustment for High/Low frequency selection. As with the B-mode image, a higher frequency offers higher strain image resolution, but a lower frequency will offer a better stress penetration depth.

- Color Blend - Rotary control around the periphery of the Elasto ON/OFF knob. It controls the intensity of the color display and reduces the transparency of the color overlay. A semi-transparent setting (around $26 \%$ ) will allow assessment of the spatial relationship between the strain map and the B-mode image. A higher, less transparent setting will give a stronger impression of the stiffness distribution.

\section{Image menu adjustments}

- Frame Reject is a filter that removes noisy, poor quality frames from the elastography sequence, e.g. when pre- \& post-compression frames are not correlated because of movement of the scan plane or signals are too weak. Using a lower value of 2 or 3 allows less experienced operators to gain confidence in acquiring elastograms, but increasing the value to 4 or 5 as the technique improves will result in elastograms with a higher signal-to-noise ratio.

- Noise Reject is a filter that removes noisy pixels within each frame (rejects regions where the echo signal amplitude is not strong enough for correlation - e.g. within cysts or other hypoechogenic areas). The rejected pixels leave transparent areas in the elastogram, where only the B-mode image is seen.

- Persistence setting can be used in conjunction with the frame and noise rejection controls to improve image quality. Increasing the persistence prolongs the time each frame is displayed on the screen and provides an overlap between consecutive frames that creates a more stable, but less responsive color display (reduces 'flashing').

- Density controls the line density. As with B-mode imaging, a lower line density will result in a higher frame rate with better temporal but lower spatial resolution, and vice versa. Increasing the line density will usually increase elastogram resolution when imaging a small $\mathrm{ROI}$ in a tissue with little movement.

- Frame rate provides additional control of the frame rate with high, medium and low selections. This allows the operator to adjust the frame rate of the elastography images to suit the compression speed and amplitude in order to achieve acceptable levels of strain between frames (which can be monitored from the strain graph display).

- Color Map - Elastograms are usually imaged as color maps superimposed on the B-mode image. There are several color maps to choose from and only the harder or softer areas can only be selected for color presentation. Most users apply the default color map where red is soft, yellow and green intermediate and blue is hard. The color map transparency relative to the B-mode image can be selected by adjusting the "blend" and is given on screen as a percentage. For the choice of color map, see the comments in the definition of color coding above.

- Elasticity-Dynamic range (E-dyn) (1-8) adjusts the dynamic range of the strain color map. The default value of 4 is most commonly used. With higher values, a larger proportion of the recorded strain values will be imaged as green and only the more extreme strain values will be displayed as blue (hard) or red (soft) [12].

\section{How to Use Strain Elastography}

\section{Prerequisites}

Select an appropriate transducer

Lower frequencies will allow assessment of deeper lesions and higher frequencies offer better spatial resolution. The sound attenuation can be assessed from the B-mode image: a good quality B-mode image is a prerequisite for obtaining a good quality elastogram. Additionally, even compression is required across the face of the transducer. Therefore, a transducer with a larger field of view is required for proper assessment of larger lesions. Footplate extenders as described earlier $\triangleright$ Fig. 1 can improve the uniformity of the stress field. 
Region of interest (ROI) size

Strain elastography (SE) displays the relative stiffness of tissue, so it is important to include sufficient normal or reference tissue surrounding the lesion of interest. The best image quality was recorded in phantom experiments when the 'lesion' of interest covered $25-50 \%$ of the ROI [12].

For breast imaging, the ROI should extend anterio-posteriorly from the subcutaneous fat tissue to the pectoralis muscle, excluding the thoracic cage, and the width should be adjusted to keep the lesion of interest within $25 \%$ of the ROI width [13]. In the case of a large lesion, the ROI can be placed towards the edge of the lesion, so that the surrounding normal tissue is included in the evaluation. Because strain imaging compares the relative stiffness between tissues, having several tissue types will aid in having an elastogram that is easier to interpret $>$ Fig. 2.

Care must be taken when using a tightly curved array transduc$\mathrm{er}$, since the region immediately in front of the transducer face could be subjected to more stress than the lateral portions of the sector. In this case, reducing the size of the elastography ROI sector will improve the uniformity of the strain image [11].

\section{Pre-compression}

The most likely cause for failing to achieve consistent, reproducible elastograms is the use of pre-compression that is too strong (see previous section: Strain based elastography - How does it work?). Firm pressure, creating a strong depression of the skin, is required for conventional $\mathrm{B}$-mode imaging. This is not the case for elasticity imaging. Applying sufficient gel between the probe and the skin will ensure that good contact is made with minimal pressure to avoid any substantial pre-compression (depression) of the tissues. From that start position, small repetitive displacements in order to obtain strain values of around $1 \%$ within the ROI allows estimation of tissue stiffness. The pace of applying and releasing stress to the insonified tissue should be matched to the frame rate (see Knobology section) in order to produce a stable and repeatable elastogram. When scanning deeply situated lesions, the selection of a lower frame rate and a slower pace of stress application may allow sufficient displacement between frames. A rate of around $2 \mathrm{~Hz}$ with a high frame rate setting has been shown in practice to give the best quality elastograms in phantom experiments [12].

\section{Checking reproducibility}

To assess the quality and reproducibility of the elastography image, freeze the image and review the stored cine loop frame by frame. A consistent color pattern obtained in a number of consecutive frames indicates a good reliable technique.

\section{Artifacts in Strain Imaging}

\section{Uneven stress over the face of the transducer}

A good layer of gel ensures no friction between the transducer and skin that can cause non-uniformity of the stress field. 'Heel-toe' movement of the transducer as often used to achieve strong ultrasound reflections in B-mode or good angle to flow in Doppler should be avoided. Uneven stress is more commonly encountered when using a tightly curved array, for example, in prostate studies, and is manifest as a 'lateral stiffness artifact' as described in the section "Region of interest (ROI) size".

\section{Cyst artifacts}

In cysts or regions devoid of echoes, measuring displacement between frames merely detects random noise which is displayed in different ways depending on the algorithm used by the manufacturer. However, these patterns have shown good results in the discrimination of cystic lesions from solid lesions. The two most commonly described types are the 'BGR sign' and 'bull's eye artifact'.

\section{BGR sign}

The 3-layer pattern (blue/green/red layers) seen in smaller cystic areas and shown in ₹ Fig. 3 has been called the BGR sign and is seen in some elastography systems. This pattern is considered a useful artifact since it highlights the cystic nature of the lesion and has been shown to be present even in cystic lesions with internal echogenic material [14]. Practical hint: Larger cysts are more often
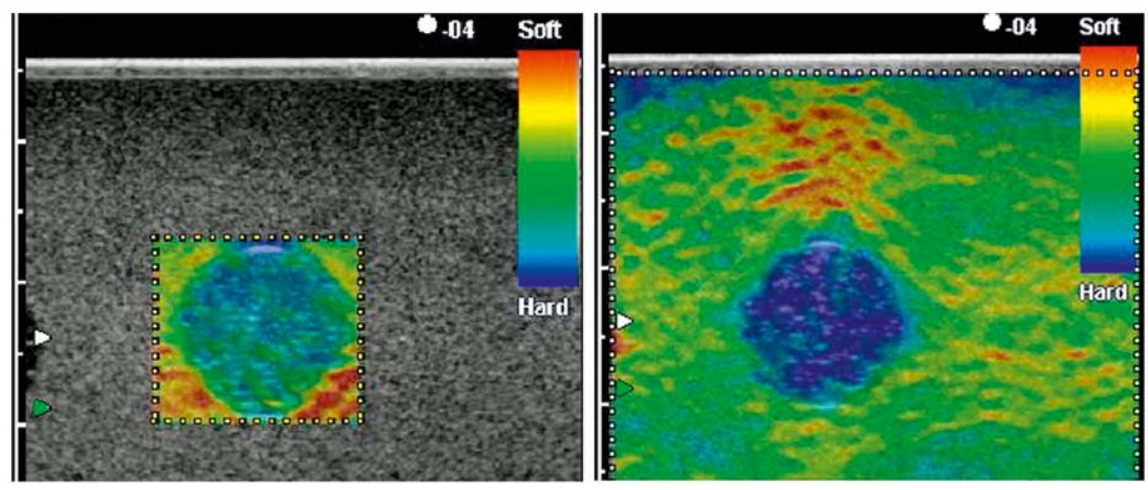

- Fig. 2 Phantom image of a hard inclusion in a softer homogeneous background. The best assessment of relative lesion stiffness is obtained when the area of interest occupies no more than $25-50 \%$ of the elastography ROI. In the right image, strain concentration is seen between the stress source (probe) and the hard lesion, indirectly confirming the presence of the harder lesion. 


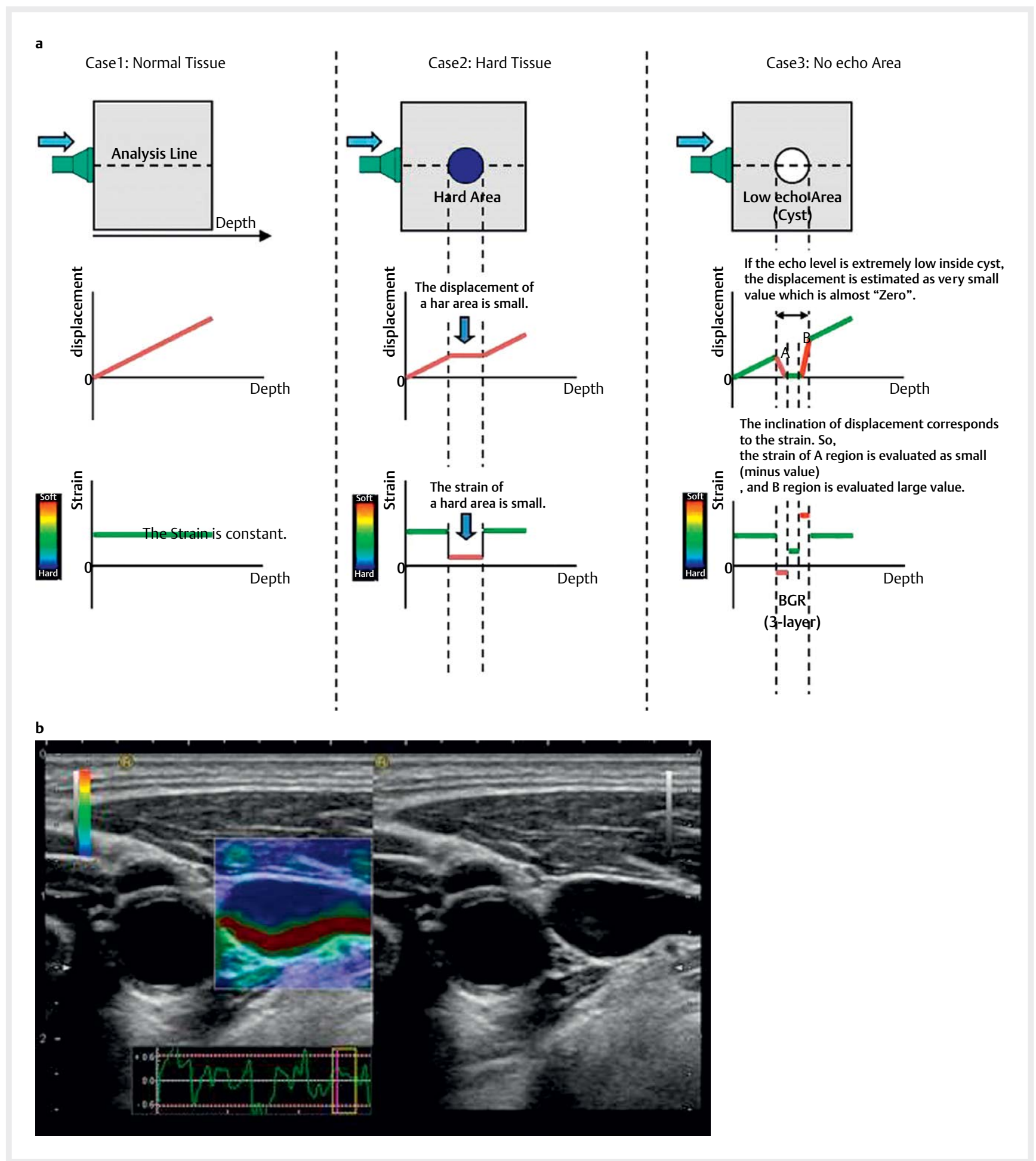

- Fig. 3 Diagrammatic explanation of the generation of the BGR artifact a. An example of the jugular vein displaying the BGR artifact $\mathbf{b}$.

seen as a 'black hole' without a strain signal, while smaller cysts in the range of $10-20 \mathrm{~mm}$ are more likely to be displayed with this artifact.

\section{Bull's eye artifact}

With other elastography systems, a "bull's eye " artifact has been described in detail [15]. Again, this artifact has shown convincing sen- sitivity and specificity in classifying benign simple and complicated cysts and can significantly decrease unnecessary breast biopsies.

\section{Stiff lesion within soft tissues}

Care should be taken to avoid assessment of tissue adjacent to stiff areas, as soft tissue will experience more strain when it is above 


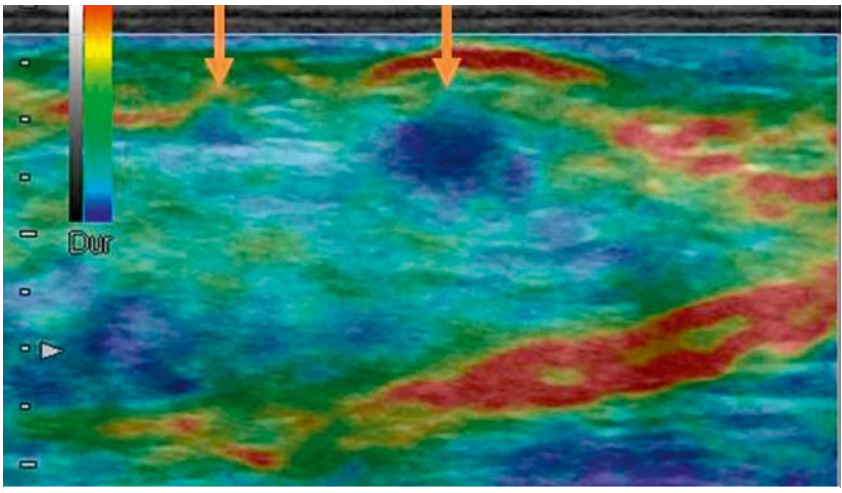

- Fig. 4 Soft tissue above stiff lesion shows more strain (red coloring) than adjacent tissue at the same depth (green coloring).

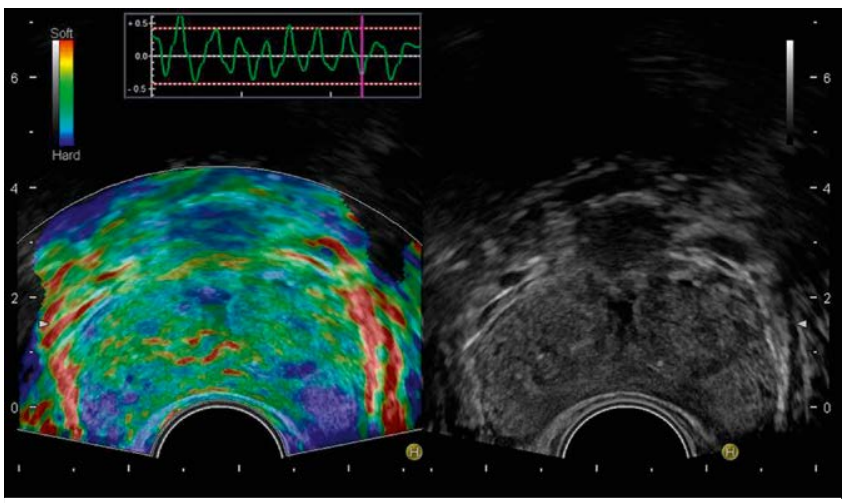

Fig. 5 'Soft rim' sign seen at the periphery of a normal prostate.

hard tissue. The differences in the appearance of soft tissue adjacent to and above a stiff lesion are shown in > Fig. 4.

\section{Slip boundaries}

Other artifacts in the elastogram can be due to tissue mobility, socalled 'slip', as seen when one region of tissue moves independently from another. These areas will be depicted red (soft), that is, large displacements are seen from one frame to the next. One such example is the 'soft rim' artifact seen in the normal prostate capsule - Fig. 5. Studies have shown that a missing 'soft rim' sign could predict extra-capsular spread of prostate disease [16-19]. Strong signals indicating "soft" tissue are also frequently seen in abdominal imaging of the pancreas and bowel, representing natural surfaces such as the peritoneum or luminal surface of the bowel.

\section{Tips and Tricks for Individual Organs}

The following provides more specific tips for applying strain-based elastography to specific organs as part of a diagnostic US examination. The discussion in this document is limited to the liver, breast, thyroid and prostate.

The transcutaneous examination technique has been described in the literature for the breast [4,20], thyroid [21-23], lymph nodes [24-29], liver [5], scrotum and many other applications including the salivary and parathyroid glands, and the musculoskeletal system $[4,30,31]$. Endoscopic ultrasound has been used to examine the pancreas [4, 32-36], lymph nodes [22, 25, 37-39], subepithelial lesions [40], anorectum [41-46], gastrointestinal tract $[31,47,48]$, endorectal ultrasound of the prostate [49], and other urogenital applications [4, 30,31]. For further reading we refer to the cited literature which also refers to much more detailed publications.

\section{Elastography in the Assessment of Breast Lesions}

\section{Introduction}

Elastography has become increasingly important in the evaluation of breast masses over the last 15 years. The fifth edition of the ACR BI-RADS Atlas, 2013, includes elasticity assessment of breast lesions as one of the associated features of ultrasound. Lesions are described as soft, intermediate or stiff. For further details on the different types of elastography that can be used for breast examinations, we recommend reading the WFUMB Guidelines and Recommendations for Clinical Use of Ultrasound Elastography: Part 2: Breast [20].

\section{Technique for performing strain elastography in the breast}

Ultrasound elastography in the evaluation of breast lesions is not a screening tool but should be performed and interpreted as an adjunct to B-mode ultrasound providing additional information to the examiner in order to make a final assessment. There is very little use for elastography in BI-RADS 1 (no lesion) or BI-RADS 2 (benign lesion) findings. In some cases, a palpable mass not visualized on B-mode ultrasound may be identified only by elastography. This is particularly true for invasive lobular cancer (ILC) and complicated cysts.

However, BI-RADS 3 and BI-RADS 4 lesions can be upgraded or downgraded using elastography. Wojcinski et al. recommended upgrading a BI-RADS 3 lesion to biopsy if the Tsukuba score is $\geq 4$ [50]. According to the WFUMB guidelines, downgrading a BIRADS 3 or 4 a lesion is considered reasonable, while downgrading a 4 b, $4 c$ or 5 lesion is not recommended.

\section{Classification methods}

After acquiring a reproducible and stable elastogram, three main diagnostic methods have been proposed to classify lesions: a visual analysis of the color pattern using the Tsukuba Score classification, semi-quantitative strain ratio and width ratio or El/B ratio.

\section{Tsukuba score/Ueno scale}

A five-point color scale, the Tsukuba score, has been described for the visual assessment of the color pattern of breast lesion stiffness relative to the background. Lesions with a higher Tsukuba score have a higher probability of malignancy. Introduced in 2006, Itoh et al. reported a sensitivity, specificity and accuracy of $86.5 \%$, $89.9 \%$ and $88.3 \%$, respectively [ 13 ]

\section{Strain ratio or fat lesion ratio}

The second possibility is the use of the strain ratio (also known as the fat lesion ratio when applied to the breast). The strain ratio is 

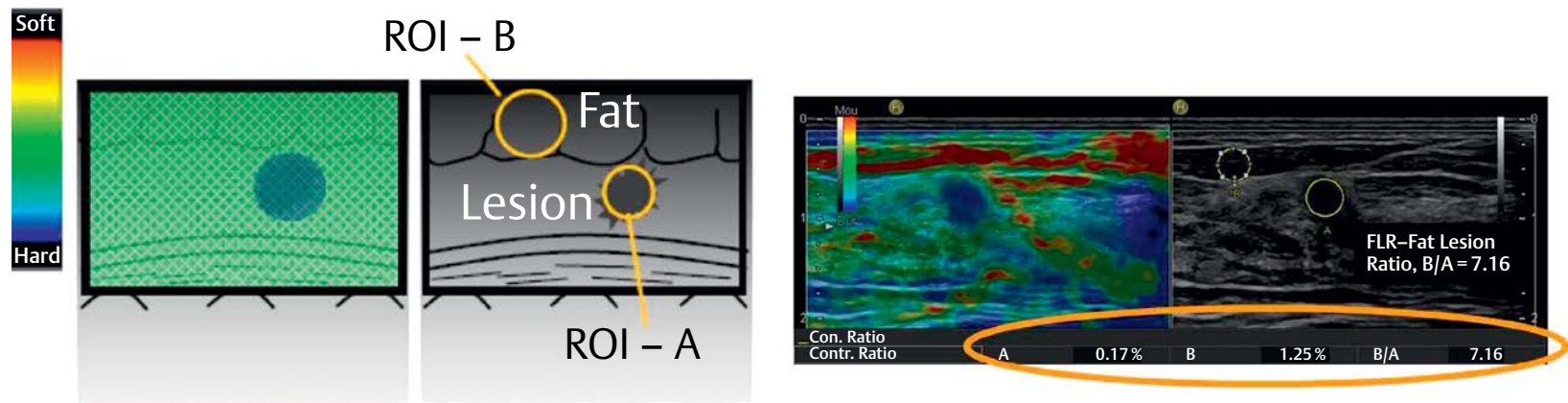

- Fig. 6 Strain ratio measurement as applied to the breast. $\mathrm{ROI} A$ is placed within the breast mass, and ROI in adjacent fat layer. Fat to lesion ratio $=\mathrm{B} / \mathrm{A}$

normally used to measure the stiffness of a discrete mass lesion. With the assumption that the stress is uniformly distributed throughout the field of view, the strain in the region of interest (ROI) can be compared to an ROI in the reference tissue that is experiencing a similar stress. This provides a semi-quantitative measurement of the relative rather than absolute tissue stiffness > Fig. 6 .

First, an ROI that best circumscribes the inside of the lesion (A) is selected and positioned. Secondly, the reference area (B) is sized and positioned in the surrounding fatty tissue. The mean strain of both of these areas is expressed as a percentage (\%) and the strain ratio (SR) is calculated as the mean strain in the reference $(B)$ divided by the mean strain in the "lesion" $(A)$ [38].

Strain ratio $(B / A)=$ Mean strain of fat area $(B) /$ mean strain in lesion of interest $(A)$

Both ROIs should be placed at the same depth. Higher strain ratio values are suspicious for malignancy. In a prospective setting Farrokh et al. reported a sensitivity and specificity of $94.4 \%$ and $87.3 \%$, respectively, with a cut-off $>2.9$ using this method [51]. There are different studies reporting different cut-off values (strain ratio 2.27-4.5) due to differences in the placement of the ROI [52]. Strain ratio measurements have also been used to classify focal lesions in other organs such as the pancreas and the thyroid $[33,35,36,53,54]$.

\section{What to avoid?}

- For the strain ratio the reference ROI should be positioned at a similar distance from the transducer surface as the lesion [55] and not placed directly above or beneath the lesion as soft tissue will experience greater strain when it is adjacent to hard tissue. In some situations, such as using the measurement as a fat-to-lesion ratio in the breast, this recommendation cannot be followed fully since the reference region is inevitably more superficial. Nevertheless, using the same positioning strategy for all measurements represents a reproducible strain index measurement for breast lesions.

- Visible blood vessels should be avoided in the ROI as the movement of blood gives an artificial effect of large displacement or 'softness'.

- Tissue boundaries that allow normal tissue movement, such as pleura, peritoneum and bowel lumen should be avoided in the ROI as slippery boundaries between tissues may show high strain (red color) as well as low strain (blue color) beyond the boundary (using a map where red is soft and blue is hard).

- If the lesion shows a heterogeneous elastogram, as a general rule, the lesion ROI should include as much of the lesion as possible in order to measure the mean stiffness of the lesion. However, the examiner must sometimes select a relevant section of the tumor, as heterogeneity may be caused by necrosis, vessels or be due to inhomogeneous stress distribution. Therefore, the observer should interpret the elastogram.

\section{Width ratio or El/B ratio}

It is known that malignant lesions appear larger on elastograms than on B-mode images. Measuring the maximum diameter of a lesion on both the B-mode image and the corresponding elastogram allows calculation of the width ratio or El/B ratio (El = elastography image size, $\mathrm{B}=\mathrm{B}$-mode size). A multicenter study analyzing 635 lesions reported a sensitivity and specificity of $99 \%$ and $87 \%$, respectively using an EI/B ratio of $<1.0$ for benign lesions and $\geq 1.0$ for malignant lesions $[56,57]$. The rationale behind this ratio is related to the invasive nature of the breast tumor [58]. Desmofibrotic reaction in the surrounding tissue has to be encountered as well.

\section{Classification of Axillary Lymph Nodes in Breast Cancer Patients}

B-mode ultrasound has a good specificity but a low sensitivity when it comes to differentiating between malignant and benign lymph nodes in breast cancer patients prior to treatment. In a retrospective study of 429 axillary ultrasound examinations of breast cancer patients, Koehler et al. reported a sensitivity of $53.6 \%$ and a specificity of $75.5 \%$ for conventional B-mode ultrasound [59]. With this background, studies have evaluated whether elastography has the potential to increase the accuracy of lymph node assessment prior to surgery.

In the literature, Wojcinski et al. reported a sensitivity of $60 \%$ and a specificity of $79.6 \%$ for strain elastography. The main feature they concentrated on was the stiffness of the cortex [60]. A recent publication by Park et al. showed that strain elastography is not able to improve diagnostic accuracy compared to B-mode ultrasound. The AUC reached 62 \% for strain elastography and $93 \%$ for conven- 
tional ultrasound. The authors used a black-gray scale elastogram and analyzed the proportions of black (hard) areas. There was no significant difference between benign and malignant nodes. Furthermore, they reported that the elastogram/B-mode size ratio was not different in these two groups [61].

All in all, elastography in the assessment of axillary lymph nodes in breast cancer patients still needs to be evaluated in further studies. Most of all, the lack of measurement standardization and the different elastography techniques must be addressed.

\section{Thyroid}

\section{Introduction}

Ultrasound elastography can be performed easily in the thyroid gland due to its superficial location. However, it is not yet used commonly in clinical practice. Rates of ultrasound elastography acceptance are higher in Europe and Asia than in the United States. In Europe and Asia, ultrasound elastography is commonly performed on thyroid nodules. For further details on the different types of elastography that can be used for thyroid studies, we recommend reading the WFUMB Guidelines and Recommendations on the Clinical Use of Ultrasound Elastography [62] and the current state-of-theart papers [63].

\section{Technique for performing strain elastography}

The general rules about performing strain elastography as described above also apply to the thyroid with some specific rules outlined below. The patient should be placed in a supine position with the neck hyperextended. A pillow or rolled up towel placed behind the neck will help with the hyperextension. Minimal pressure should be applied to the neck while acquiring images. The patient should then be asked to hold his/her breath while the images are acquired. Care should be taken to place the focal zone at or below the level of the nodule. If the strain ratio measurement is to be obtained, part of the normal thyroid or adjacent muscle should be included in the image. Isthmus nodules may be difficult to assess due to their superficial location and the possibility of inducing near-field artifacts [64]. Accurate measurement of deeply located nodules may not be possible due to the depth-related decay of the stress [65].

Areas of dense calcification should be avoided since these areas tend to have higher stiffness which may not be representative of the surrounding soft tissue nodule [66]. Similarly, regions of interest should not be placed posterior to cystic areas due to the likely artifactual increase in stiffness seen posterior to the cystic area. Representative images should be taken from multiple sites in the nodule. If the nodule is small, multiple images should be taken at the same location. Between 5 and 10 measurements should be acquired for measurement of the stiffness and the average value obtained.

\section{Classification methods}

Visual scoring systems: A modification of the Tsukuba scoring system for the breast was revised for use in the thyroid. Studies have found a sensitivity of $97 \%$, specificity of $100 \%$, positive predictive value of $100 \%$, and negative predictive value of $98 \%[67,68]$. A 4-point scoring system used by Asteria et al. showed sensitivities and specificities of US elastography for thyroid cancer diagnosis of $94.1 \%$ and $81 \%$ with positive and negative predictive values of $55.2 \%$ and $98.2 \%$, respectively [69].

Strain ratio: Two types of strain ratio have been defined: parenchyma-to-nodule strain ratio (PNSR), which is the mean strain in the normal adjacent thyroid parenchyma divided by the mean strain within the thyroid nodule, and the muscle-to-nodule strain ratio (MNSR), which is the mean strain in an adjacent strap muscle divided by the strain in the nodule [70]. MNSR is used when a nodule is large and there is no adjacent normal thyroid parenchyma with which to compare the strain. No significant difference was found between PNSR and MNSR in the distinction between malignant and benign nodules and hence either one can be used depending on the size and location of the nodule [71]. Several studies have evaluated the strain ratio. However, there is no agreement on the best cut-off point to differentiate between benign and malignant nodules [72-77].

When using the pulsation of the carotid artery as the compression source on the thyroid, a semi-quantitative approach similar to strain ratio, known as the elasticity contrast index, can be used [78].

\section{How to implement strain elastography in clinical practice}

Ultrasound elastography is not recommended as a screening tool at present for the differentiation of benign from malignant thyroid nodules. Preliminary ultrasound elastography results come to different conclusions about the identification of malignant nodules, and some malignancies are difficult to identify on ultrasound elastography alone. A study by Xue et al. has shown that the combination of TI-RADS and ultrasound elastography had a higher diagnostic sensitivity and accuracy for the differentiation of malignant thyroid nodules than using either method alone [79]. As discussed by Cosgrove et al. in the WFUMB guidelines paper, a significant number of pitfalls and practical issues have to be considered before interpreting the results of ultrasound elastography and using them in clinical practice [23].

\section{Prostate}

\section{Introduction}

Prostate cancer has the highest incidence rate and is the second highest cause of cancer death in men in western countries. The screening standard has been a combination of digital rectal exam (DRE) and prostate-specific antigen (PSA). An abnormality is an indication for transrectal ultrasound (TRUS)-guided biopsy. There are two major drawbacks of this screening method. First, a substantial number of unnecessary biopsies with no or indolent cancer with false-positive results are common ( $>50 \%$ ), and second, $20 \%$ of prostate cancers will have a normal PSA.

Ultrasound is the most common imaging modality for the evaluation of the prostate due to its real-time imaging, does not involve ionizing radiation and is low in cost. However, TRUS is not highly sensitive or specific (40-50\%) in the diagnosis of prostate cancer. Both strain (SE) and shear wave elastography (SWE) have been used to improve lesion detection and the characterization of prostate lesions. 


\section{Technique for performing strain elastography in the prostate}

A detailed discussion on how to perform prostate elastography can be found in the WFUMB guidelines [80]. A brief review is provided here.

The principles of strain elastography discussed above are applicable to prostate elastography. However, one problem of strain elastography is applying uniform stress over the prostate using a curved endorectal transducer to achieve reproducible strain (displacement). A water-filled balloon can be placed between the transducer and the rectal wall to allow for more homogeneous displacement.

No specific preparation is required for transrectal strain elastography. A standard B-mode prostate exam including the seminal vessels, the base, mid and apex of the prostate and some surrounding tissues should be performed. Color or power Doppler including the whole gland with attention to any hypoechoic nodules in the peripheral gland should also be performed. After the standard exam, elastography should be performed from the base to the apex of the gland. The SE technique should include minimal pre-compression, placement of the focal zone in the far field of the region of interest, having a field of view size to include the prostate capsule and some periprostatic tissues excluding the bladder. Keeping the area of concern in the center of the field of view will provide improved elastograms. It is generally accepted that there is a learning curve when performing SE of the prostate.

Several methods of interpretation have been proposed. No uniformly accepted scoring system has been established. A five-point subjective scale has been proposed by Kamoi [81]. That scoring system is as follows, assuming that a color scale where blue is stiff and red is soft is used:

1. Score 1: Normal. Homogeneous strain, the entire gland is evenly shaded in green

2. Score 2: Probably normal. Symmetric heterogeneous strain, the gland is a mosaic pattern of green and blue

3. Score 3: Indeterminate. Focal asymmetric lesion without strain (stiff) not related to a hypo-echoic lesion

4. Score 4: Probably carcinoma. Strain at the periphery of the hypoechoic lesion on B-mode with sparing of the center of the lesion, the peripheral part of the lesion is green and the central part is blue

5. Score 5: Definitely carcinoma. No strain in the entire hypoechoic lesion on B-mode or in the surrounding area, the entire lesion is blue

Using a cut-off value of 3 , this technique had a sensitivity of $68 \%$, a specificity of $81 \%$ and an accuracy of $76 \%$ [81].

Another scoring system has been proposed by Xu et al. [82]. This system has the ROI set to approximately $1 \mathrm{~cm}$ at the edge of the largest transverse image. The color-coded system uses blue as stiff and red as soft and is as follows:

- Score 1: No blue area or star-like blue in the peripheral gland

- Score 2: Mosaic or a small symmetric blue area bilaterally in the outer gland (blue area $<5 \mathrm{~mm}$ )

- Score 3: A small symmetric blue area in the outer gland (blue area $=>5 \mathrm{~mm}$ )
- Score 4: Asymmetric blue area in the outer gland (blue area $=>5 \mathrm{~mm}$ )

- Score 5: Asymmetric blue area in the outer gland (blue area $=>50 \%$ of single outer gland area)

With this scoring method, the area under the curve (AUC) is 0.729 with the best cut-off value score of 3 . This method yielded a sensitivity of $68.6 \%$, specificity of $69.4 \%$ and an accuracy of $69.2 \%$. Regardless of the scoring system used, the SE should not be interpreted without considering the B-mode findings.

Several papers have evaluated the SE of the prostate for the detection of prostate cancer [80]. The sensitivity of these studies ranged from $49 \%$ to $87 \%$ with specificities of $60-92 \%$. It is generally accepted that the addition of SE will increase the prostate cancer detection rate and the positive biopsy rate compared to TRUS, that the technique is more accurate in larger cancers and in higher Gleason score lesions, and that targeted biopsies should always be performed with standard systematic biopsies. It is well documented that the presence of calcifications can cause false-positive results.

\section{How to implement strain elastography in clinical practice}

The addition of strain elastography to the standard TRUS exam is not difficult. The ultrasound system must have strain available on the endorectal transducer. It only adds a few minutes of extra time to the exam. There is a learning curve to obtain optimal strain elastograms. The ability to biopsy lesions under direct elastographic guidance should be available and performed in addition to the standard systematic biopsies. Ideally, having the ability to perform these biopsies with US/mpMRI fusion would optimize cancer detection with biopsy of both the lesions detected on SE as well as the mpMRI lesions.

\section{Tips \& tricks}

- Apply uniform stress with light pressure on the prostate

- Optimal elastograms are usually obtained when keeping the area of evaluation centered in the FOV

- Using a water balloon between the transducer and rectal wall can aid in applying more uniform pressure

\section{Liver Elastography}

\section{Introduction}

The liver is a large parenchymal organ readily accessible to US scanning including elasticity imaging and elastography measurements. Elastography may be used to describe the stiffness contrast between a focal liver lesion and the surrounding parenchyma, but of more clinical importance, it can be used to describe the increased stiffness when the liver becomes fibrotic as a result of chronic diffuse liver disease. From the mosaic color pattern obtained with elastography, features can be derived for quantitative evaluation and calculation of a liver fibrosis index (LFI) has been proposed. This index is based on a histogram distribution of strains recorded in the selected field of view as described below. 


\section{Technique for performing strain elastography in the liver}

For liver fibrosis analysis, using an intercostal space as a window, the transducer can be directed towards the heart so that the strain image is generated by the pressure exerted on the liver by cardiac movement. The patient should stop breathing in mid-inspiration/ expiration during the acquisition. It might be difficult to obtain a representative histogram in obese patients or in patients who cannot hold their breath sufficiently well.

\section{How to implement strain elastography in clinical practice}

Strain imaging of the liver can easily be implemented in the workflow of liver examinations when focal liver lesions are evaluated as well as in diffuse liver disease, such as hepatitis or toxic/alcoholic liver disease. After scanning the liver in B-mode, use of color Doppler to visualize portal and hepatic veins and even pulsed Doppler for measurement of flow velocities, the elastography mode may be used. Areas in both the left and right liver can be selected using strain elastography. Patients may need to hold their breath in the mid-inspiratory phase, but when scanning the left lobe, cardiac movements may create larger measurement variability in the left compared to the right liver lobe. When focal lesions are seen, a section where the lesion occupies $25-50 \%$ of the ROI may be selected and a loop of approximately 5 seconds providing a repetitive "stable" elastogram. Then the frames with maximum negative strains are preferred for measuring 3-5 separate strain ratios or strain histograms. In diffuse liver disease using the strain histogram and LFI index (see separate section on $\mathrm{LFI}$ ) is an option. It is recommended to repeat the measurements in 5-10 frames to provide a median value. Some scanners can also be set to average the measurements over 1-3 compressions. In diffuse liver disease, most users apply elastography imaging and measurements intercostally to the right liver lobe. In clinical practice, a mere visual evaluation can also provide valuable feedback to the examining physician without measurements. Measurements or stored images provide better documentation.

\section{What to avoid?}

- Large blood vessels should be avoided in the ROI as movement of blood gives an artificial effect of large displacement or 'softness'

- For liver fibrosis assessment, the elastography ROI should be placed at least $1 \mathrm{~cm}$ below the liver capsule to avoid the region of low strain (blue coloring) below the capsule

\section{Classification method - liver fibrosis index}

From the mosaic pattern typically seen in diffuse liver disease, the distribution of recorded strains within a region of interest can be displayed as a histogram from which a number of statistical parameters can be derived for quantitative evaluation. The key parameters (features extracted from the strain image) are: mean strain (MEAN); standard deviation of the mean (SD); the percentage of blue area (\% AREA); complexity of the blue areas (COMP) (relation between the circumference and the area of blue patches). The shape of the histogram described mathematically by skewness and kurtosis also reflects the distribution and tells us something about the homogeneity or otherwise of the tissue stiffness recorded.

The histograms can be displayed on the screen along with the abovementioned parameters from which the liver fibrosis index has been derived using multiple regression analysis [83-85]:

LF Index $=-0.00897 \times$ MEAN $-0.00502 \times$ SD $+0.0232 \times \%$ AREA + $0.0253 \times$ COMP $+0.775 \times$ SKEW $-0.281 \times$ KURT $+2.08 \times$ ENT + $3.04 \times$ IDM + 40.0 $\times$ ASM -5.54 [83]

Strain histograms are difficult to standardize in free-hand applications as the displayed strains depend on the degree of pre-compression and stress (force) that is applied. In order to create adequate strain in the deep parts of the liver, more stress must be applied on the surface. However, provided that movements result in mean strain values of $0.1-1.0 \%$, in the elasticity ROI, the histogram will provide information on the distribution of strains within the ROI, i.e. reflecting the homogeneity as well as the tissue stiffness.

\section{Tips \& tricks}

- Have good contact with the skin

- Establish a good B-mode image

- Select the ROI at least 1-2 cm from the liver capsule

- Avoid large vessels

- Reduce pre-compression as much as possible without losing the B-mode image quality

- Record 5-10 measurements, save the image and use the median results (strain ratio, mean histogram value, LFI, \% blue area etc.)

\section{Pancreas Elastography}

Strain endoscopic ultrasound elastography shows potential for the diagnosis and differentiation of pancreatic tumors because malignant tissue tends to be harder than benign lesions and/or adjacent normal tissues [2]. According to current knowledge, SE can predict benign disease if the lesion is displayed as soft. Nevertheless, a hard lesion can be either benign or malignant.

EUS elastography shows some limitations including the non-standardized pressure using the probe and the image reconstruction always uses the complete color spectrum within the sample area. In addition, the reject function, persistence, and dynamic range could help to reduce artifacts and to simplify image acquisition, but carry a risk of distortion/reduction of image information. The limited ROI size and depth penetration prevents border delineation of lesions located too far from the transducer. A major limitation of EUS elastography is the image selection and qualitative pattern analysis, which is subjective and associated with significant intraobserver and interobserver variability.

We also refer to the recently published article describing elastography using conventional endoscopic ultrasound [1].

\section{Future Developments, Outlook}

Several versions of strain elastography have emerged from the major manufacturers of US equipment. Real-time tissue elastography (RTE) from Hitachi was the first commercially available sys- 
tem that displayed a color-coded elastogram in real time overlaying the B-mode image and some of the terminology in this manuscript is derived from this system. Most major ultrasound vendors now offer a version of strain elastography on their systems with sometimes different terminology which has not been discussed in depth in this paper. A critical discussion of terminology is needed but was not the intention of this manuscript. Strain elastography allows an overview of relative tissue stiffness in a large field of view. Other methods available for measuring and displaying tissue stiffness are based on shear wave speed. These methods have proved very useful in following the development of liver fibrosis in chronic liver disease.

Within a limited clinical scenario, such as colorectal adenoma and early carcinoma, the method has performed better than US and MRI with respect to diagnostic accuracy [43] but further studies are needed.

Elastography has created new possibilities in medical imaging, and is still in the early stages of development. It has also raised the understanding of imaging biology. There are several processes, both inflammatory and neoplastic, that serve to increase tissue stiffness, and imaging tissue hardness provides only one aspect of our understanding.

\section{Conclusion: Benefits and Limitations of Strain Elastography in Clinical Applications}

Strain-based elastography enables visualization and relative quantification of tissue stiffness in areas inaccessible to palpation. As a diagnostic tool, strain elastography has proven to be beneficial in the evaluation of focal lesions in the breast, thyroid, lymph nodes and pancreatic lesions. However, for many applications where the distinction between a benign and malignant lesion is crucial, consecutive series have shown that the specificity of the method is not satisfactory as a single modality. Strain elastography cannot replace tissue specimens, but several reports have concluded that strain elastography is most effectively used as an adjunct to B-mode US with Doppler or contrast-enhanced US. Generally, the combination of a hypoechoic lesion on B-mode with a low strain signal (hard) should raise suspicion of a malignant lesion in most organs.

Strain elastography is performed using the probe or internal tissue movements to calculate local strain. This strain is displayed as a color image. The main limitation of this method is that the applied stress is unknown. Therefore, the absolute value of the Elasticity or Young's Modulus of the tissue cannot be calculated. The method relies on an even distribution of stress over the ROI, but this is sometimes difficult to obtain in practice and the stress is attenuated (weakened) as it is transmitted through tissues. Anisotropy of tissues in vivo can cause variable stress distributions and thus variable strains. The boundaries of tissue and the movement between organs also result in challenges for this imaging method.

\section{Conflict of Interest}

The authors declare that they have no conflict of interest.
References

[1] Dietrich CF et al. How to perform endoscopic ultrasoud elastography. Endosc Ultrasound 2017 in press

[2] Bamber ], Cosgrove D, Dietrich CF, Fromageau J, Bojunga J, Calliada F, Cantisani $V$ et al. EFSUMB guidelines and recommendations on the clinical use of ultrasound elastography. Part 1: Basic principles and technology. Ultraschall Med 2013; 34: 169-184

[3] Shiina T, Nightingale KR, Palmeri ML, Hall T], Bamber JC, Barr RG, Castera $L$ et al. WFUMB guidelines and recommendations for clinical use of ultrasound elastography: Part 1: basic principles and terminology. Ultrasound Med Biol 2015; 41: 1126-1147

[4] Cosgrove D, Piscaglia F, Bamber J, Bojunga J, Correas JM, Gilja OH, Klauser AS et al. EFSUMB guidelines and recommendations on the clinical use of ultrasound elastography. Part 2: Clinical applications. Ultraschall Med 2013; 34: 238-253

[5] Ferraioli G, Filice C, Castera L, Choi BI, Sporea I, Wilson SR, Cosgrove D et al. WFUMB guidelines and recommendations for clinical use of ultrasound elastography: Part 3: liver. Ultrasound Med Biol 2015; 41: $1161-1179$

[6] Dong Y, Sirli R, Ferraioli G, Sporea I, Chiorean L, Cui X, Fan M et al. Shear wave elastography of the liver - review on normal values. Z Gastroenterol 2017; 55: 153-166

[7] Dietrich CF, Bamber ], Berzigotti A, Bota S, Cantisani V, Castera L, Cosgrove D et al. EFSUMB Guidelines and recommendations on the clinical use of liver ultrasound elastography, Update 2017 (Long Version). Ultraschall Med 2017; 38: e16-e47

[8] Dietrich CF, Bamber J, Berzigotti A, Bota S, Cantisani V, Castera L, Cosgrove $\mathrm{D}$ et al. EFSUMB Guidelines and recommendations on the clinical use of liver ultrasound elastography, Update 2017 (Short Version). Ultraschall Med 2017; 38: 377-394

[9] Cui XW, Friedrich-Rust M, De Molo C, Ignee A, Schreiber-Dietrich D, Dietrich CF. Liver elastography, comments on EFSUMB elastography guidelines 2013. World J Gastroenterol 2013; 19: 6329-6347

[10] Barr RG, Zhang Z. Effects of precompression on elasticity imaging of the breast: development of a clinically useful semiquantitative method of precompression assessment. J Ultrasound Med 2012; 31: 895-902

[11] Bilgen M, Insana MF. Error analysis in acoustic elastography. II. Strain estimation and SNR analysis. J Acoust Soc Am 1997; 101: 1147-1154

[12] Havre RF, Elde E, Gilja OH, Odegaard S, Eide GE, Matre K, Nesje LB. Freehand real-time elastography: impact of scanning parameters on image quality and in vitro intra- and interobserver validations. Ultrasound Med Biol 2008; 34: 1638-1650

[13] Itoh A, Ueno E, Tohno E, Kamma H, Takahashi H, Shiina T, Yamakawa M et al. Breast disease: clinical application of US elastography for diagnosis. Radiology 2006; 239: 341-350

[14] Cho N, Moon WK, Chang JM, Kim S], Lyou CY, Choi HY. Aliasing artifact depicted on ultrasound (US)-elastography for breast cystic lesions mimicking solid masses. Acta Radiol 2011; 52: 3-7

[15] Barr RG, Lackey AE. The utility of the "bull's-eye" artifact on breast elasticity imaging in reducing breast lesion biopsy rate. Ultrasound $Q$ 2011; 27: 151-155

[16] Pallwein L, Mitterberger M, Struve P, Pinggera G, Horninger W, Bartsch $\mathrm{G}$, Aigner $\mathrm{F}$ et al. Real-time elastography for detecting prostate cancer: preliminary experience. BJU Int 2007; 100: 42-46

[17] Pallwein L, Aigner F, Faschingbauer R, Pallwein E, Pinggera G, Bartsch $G$, Schaefer $G$ et al. Prostate cancer diagnosis: value of real-time elastography. Abdom Imaging 2008; 33: 729-735

[18] Aigner F, Pallwein L, Junker D, Schafer G, Mikuz G, Pedross F, Mitterberger MJ et al. Value of real-time elastography targeted biopsy for prostate cancer detection in men with prostate specific antigen 1.25 $\mathrm{ng} / \mathrm{ml}$ or greater and $4.00 \mathrm{ng} / \mathrm{ml}$ or less. J Urol 2010; 184: 913-917 
[19] Aigner F, Pallwein L, Schocke M, Lebovici A, Junker D, Schafer G, Mikuz $G$ et al. Comparison of real-time sonoelastography with T2-weighted endorectal magnetic resonance imaging for prostate cancer detection. J Ultrasound Med 2011; 30: 643-649

[20] Barr RG, Nakashima K, Amy D, Cosgrove D, Farrokh A, Schafer F, Bamber JC et al. WFUMB guidelines and recommendations for clinical use of ultrasound elastography: Part 2: breast. Ultrasound Med Biol 2015; 41: 1148-1160

[21] Friedrich-Rust M, Vorlaender C, Dietrich CF, Kratzer W, Blank W, Schuler A, Broja $\mathrm{N}$ et al. Evaluation of strain elastography for differentiation of thyroid nodules: Results of a prospective DEGUM multicenter study. Ultraschall Med 2016; 37: 262-270

[22] Dietrich CF, Bojunga J. Ultrasound of the thyroid. Z Gastroenterol 2015; 53: 208-225

[23] Cosgrove D, Barr R, Bojunga J, Cantisani V, Chammas MC, Dighe M, Vinayak $S$ et al. WFUMB Guidelines and recommendations on the clinical use of ultrasound elastography: Part 4. Thyroid. Ultrasound Med Biol 2016

[24] Chiorean L, Barr RG, Braden B, Jenssen C, Cui XW, Hocke M, Schuler A et al. Transcutaneous ultrasound: Elastographic lymph node evaluation. Current clinical applications and literature review. Ultrasound Med Biol 2016; 42: 16-30

[25] Havre RF, Leh SM, Gilja OH, Odegaard S, Waage JE, Baatrup G, Nesje LB. Differentiation of metastatic and non-metastatic mesenteric lymph nodes by strain elastography in surgical specimens. Ultraschall Med 2016

[26] Cui XW, Hocke M, Jenssen C, Ignee A, Klein S, Schreiber-Dietrich D, Dietrich CF. Conventional ultrasound for lymph node evaluation, update 2013. Z Gastroenterol 2014; 52: 212-221

[27] Cui XW, Jenssen C, Saftoiu A, Ignee A, Dietrich CF. New ultrasound techniques for lymph node evaluation. World J Gastroenterol 2013; 19 : 4850-4860

[28] Dietrich CF, Hocke M, Jenssen C. Ultrasound for abdominal lymphadenopathy. Dtsch Med Wochenschr 2013; 138: 1001-1018

[29] Dietrich CF, Ponnudurai R, Bachmann Nielsen M. Is there a need for new imaging methods for lymph node evaluation? Ultraschall Med 2012; 33: 411-414

[30] Dietrich CF. Echtzeit-Gewebeelastographie. Anwendungsmöglichkeiten nicht nur im Gastrointestinaltrakt. Endheu 2010; 23: 177-225

[31] Dietrich CF. Multiple clinical applications. Multiple clinical solutions. Endheu 2012; 24: 177-225

[32] Dietrich CF, Hirche TO, Ott M, Ignee A. Real-time tissue elastography in the diagnosis of autoimmune pancreatitis. Endoscopy 2009; 41: $718-720$

[33] Hirche TO, Ignee A, Barreiros AP, Schreiber-Dietrich D, Jungblut S, Ott $\mathrm{M}$, Hirche $\mathrm{H}$ et al. Indications and limitations of endoscopic ultrasound elastography for evaluation of focal pancreatic lesions. Endoscopy 2008; 40: 910-917

[34] Saftoiu A, Vilmann P, Gorunescu F, Janssen J, Hocke M, Larsen M, Iglesias-Garcia J et al. Efficacy of an artificial neural network-based approach to endoscopic ultrasound elastography in diagnosis of focal pancreatic masses. Clin Gastroenterol Hepatol 2012; 10: 84-90 e81

[35] Saftoiu A, Vilmann P, Gorunescu F, Janssen J, Hocke M, Larsen M, Iglesias-Garcia J et al. Accuracy of endoscopic ultrasound elastography used for differential diagnosis of focal pancreatic masses: A multicenter study. Endoscopy 2011; 43: 596-603

[36] Havre RF, Odegaard S, Gilja OH, Nesje LB. Characterization of solid focal pancreatic lesions using endoscopic ultrasonography with real-time elastography. Scand J Gastroenterol 2014; 49: 742-751

[37] Cui XW, Chang JM, Kan QC, Chiorean L, Ignee A, Dietrich CF. Endoscopic ultrasound elastography: Current status and future perspectives. World J Gastroenterol 2015; 21: 13212-13224
[38] Dietrich CF, Saftoiu A, Jenssen C. Real time elastography endoscopic ultrasound (RTE-EUS), a comprehensive review. Eur J Radiol 2014; 83: 405-414

[39] Janssen J, Dietrich CF, Will U, Greiner L. Endosonographic elastography in the diagnosis of mediastinal lymph nodes. Endoscopy 2007; 39: 952-957

[40] Dietrich CF, Jenssen C, Hocke M, Cui XW, Woenckhaus M, Ignee A. Imaging of gastrointestinal stromal tumours with modern ultrasound techniques - a pictorial essay. Z Gastroenterol 2012; 50: 457-467

[41] Waage JE, Bach SP, Pfeffer F, Leh S, Havre RF, Odegaard S, Baatrup G. Combined endorectal ultrasonography and strain elastography for the staging of early rectal cancer. Colorectal Dis 2015; 17: 50-56

[42] Waage JE, Havre RF, Odegaard S, Leh S, Eide GE, Baatrup G. Endorectal elastography in the evaluation of rectal tumours. Colorectal Dis 2011; 13: $1130-1137$

[43] Waage JE, Leh S, Rosler C, Pfeffer F, Bach SP, Havre RF, Haldorsen IS et al. Endorectal ultrasonography, strain elastography and MRI differentiation of rectal adenomas and adenocarcinomas. Colorectal Dis 2015; 17: 124-131

[44] Waage JE, Rafaelsen SR, Borley NR, Havre RF, Gubberud ET, Leh S, Kolbro T et al. Strain elastography evaluation of rectal tumors: interand intraobserver reproducibility. Ultraschall Med 2015; 36: 611-617

[45] Allgayer H, Ignee A, Dietrich CF. Endosonographic elastography of the anal sphincter in patients with fecal incontinence. Scand J Gastroenterol 2010; 45: 30-38

[46] Allgayer H, Ignee A, Zipse S, Crispin A, Dietrich CF. Endorectal ultrasound and real-time elastography in patients with fecal incontinence following anorectal surgery: A prospective comparison evaluating short- and long-term outcomes in irradiated and non-irradiated patients. Z Gastroenterol 2012; 50: 1281-1286

[47] Havre R, Gilja $\mathrm{OH}$. Elastography and strain rate imaging of the gastrointestinal tract. Eur J Radiol 2014; 83: 438-441

[48] Havre RF, Leh S, Gilja OH, Odegaard S, Waage JE, Baatrup G, Nesje LB. Strain assessment in surgically resected inflammatory and neoplastic bowel lesions. Ultraschall Med 2014; 35: 149-158

[49] Barr RG, Cosgrove D, Brock M, Cantisani V, Correas JM, Postema AW, Salomon G et al. WFUMB Guidelines and recommendations on the clinical use of ultrasound elastography: Part 5. Prostate. Ultrasound Med Biol 2016

[50] Wojcinski S, Boehme E, Farrokh A, Soergel P, Degenhardt F, Hillemanns P. Ultrasound real-time elastography can predict malignancy in BI-RADS(R)-US 3 lesions. BMC Cancer 2013; 13: 159

[51] Farrokh A, Wojcinski S, Degenhardt F. Diagnostic value of strain ratio measurement in the differentiation of malignant and benign breast lesions. Ultraschall Med 2011; 32: 400-405

[52] Sadigh G, Carlos RC, Neal CH, Dwamena BA. Accuracy of quantitative ultrasound elastography for differentiation of malignant and benign breast abnormalities: a meta-analysis. Breast Cancer Res Treat 2012; 134: 923-931

[53] Dighe M, Bae U, Richardson ML, Dubinsky T], Minoshima S, Kim Y. Differential diagnosis of thyroid nodules with US elastography using carotid artery pulsation. Radiology 2008; 248: 662-669

[54] Friedrich-Rust M, Sperber A, Holzer K, Diener ], Grunwald F, Badenhoop K, Weber $S$ et al. Real-time elastography and contrast-enhanced ultrasound for the assessment of thyroid nodules. Exp Clin Endocrinol Diabetes 2010; 118: 602-609

[55] Havre RF, Waage JR, Gilja OH, Odegaard S, Nesje LB.Real-Time elastography strain ratio measurements are influenced by the position of the reference area. Ultraschall Med. 2012; 33: 559-568

[56] Barr RG, Destounis S, Lackey LB 2nd, Svensson WE, Balleyguier C, Smith C. Evaluation of breast lesions using sonographic elasticity imaging: A multicenter trial. J Ultrasound Med 2012; 31: 281-287 
[57] Barr RG, Zhang Z. Shear-wave elastography of the breast: value of a quality measure and comparison with strain elastography. Radiology 2015; 275: 45-53

[58] Grajo JR, Barr RG. Strain elastography for prediction of breast cancer tumor grades. J Ultrasound Med 2014; 33: 129-134

[59] Koehler KE, Ohlinger R. Sensitivity and specificity of preoperative ultrasonography for diagnosing nodal metastases in patients with breast cancer. Ultraschall Med 2011; 32: 393-399

[60] Wojcinski S, Dupont J, Schmidt W, Cassel M, Hillemanns P. Real-time ultrasound elastography in 180 axillary lymph nodes: Elasticity distribution in healthy lymph nodes and prediction of breast cancer metastases. BMC Med Imaging 2012; 12: 35

[61] Park YM, Fornage BD, Benveniste AP, Fox PS, Bassett RL Jr., Yang WT. Strain elastography of abnormal axillary nodes in breast cancer patients does not improve diagnostic accuracy compared with conventional ultrasound alone. AJR Am J Roentgenol 2014; 203: 1371-1378

[62] Cosgrove D, Barr R, Bojunga J, Cantisani V, Chammas MC, Dighe M, Vinayak $S$ et al. WFUMB Guidelines and Recommendations on the Clinical Use of Ultrasound Elastography: Part 4. Thyroid. Ultrasound Med Biol 2017; 43: 4-26

[63] Dighe M, Barr R, Bojunga J, Cantisani V, Chammas MC, Cosgrove D, Cui XW et al. Thyroid Ultrasound: State of the Art Part 1 - Thyroid Ultrasound reporting and Diffuse Thyroid Diseases. Med Ultrason 2017; 19: 79-93

[64] Wang Y, Dan H], Dan HY, Li T, Hu B. Differential diagnosis of small single solid thyroid nodules using real-time ultrasound elastography. J Int Med Res 2010; 38: 466-472

[65] Tranquart F, Bleuzen A, Pierre-Renoult P, Chabrolle C, Sam Giao M, Lecomte P. Elastosonography of thyroid lesions. J Radiol 2008; 89: 35-39

[66] Shuzhen C. Comparison analysis between conventional ultrasonography and ultrasound elastography of thyroid nodules. Eur J Radiol 2012; 81: 1806-1811

[67] Rago T, Vitti P. Role of thyroid ultrasound in the diagnostic evaluation of thyroid nodules. Best Pract Res Clin Endocrinol Metab 2008; 22: 913-928

[68] Rago T, Di Coscio G, Basolo F, Scutari M, Elisei R, Berti P, Miccoli P et al. Combined clinical, thyroid ultrasound and cytological features help to predict thyroid malignancy in follicular and Hupsilonrthle cell thyroid lesions: results from a series of 505 consecutive patients. Clin Endocrinol (Oxf) 2007; 66: 13-20

[69] Asteria C, Giovanardi A, Pizzocaro A, Cozzaglio L, Morabito A, Somalvico F, Zoppo A. US-elastography in the differential diagnosis of benign and malignant thyroid nodules. Thyroid 2008; 18: 523-531

[70] Ciledag N, Arda K, Aribas BK, Aktas E, Köse SK. The utility of ultrasound elastography and MicroPure imaging in the differentiation of benign and malignant thyroid nodules. AJR Am J Roentgenol 2012; 198 : W244-W249

[71] Aydin R, Elmali M, Polat AV, Danaci M, Akpolat I. Comparison of muscle-to-nodule and parenchyma-to-nodule strain ratios in the differentiation of benign and malignant thyroid nodules: Which one should we use? Eur ] Radiol 2014; 83: e131-E136
[72] Cantisani V, Grazhdani H, Drakonaki E et al. HYPERLINK "https://www. ncbi.nlm.nih.gov/pubmed/25954310" Strain US Elastography for the Characterization of Thyroid Nodules: Advantages and Limitation. Int J Endocrinol 2015; doi: 10.1155/2015/908575

[73] Cantisani V, Lodise P, Grazhdani H et al. HYPERLINK "https://www.ncbi. nlm.nih.gov/pubmed/23763859" Ultrasound elastography in the evaluation of thyroid pathology. Current status. Eur J Radiol 2014; 83 : 420-428. doi: 10.1016/j.ejrad.2013.05.008

[74] Ding J, Cheng H, Ning C, Huang J, Zhang Y. Quantitative measurement for thyroid cancer characterization based on elastography. J Ultrasound Med 2011; 30: 1259-1266

[75] Xing P, Wu L, Zhang C, Li S, Liu C, Wu C. Differentiation of benign from malignant thyroid lesions: Calculation of the strain ratio on thyroid sonoelastography. J Ultrasound Med 2011; 30: 663-669

[76] Kagoya R, Monobe H, Tojima H. Utility of elastography for differential diagnosis of benign and malignant thyroid nodules. Otolaryngol Head Neck Surg 2010; 143: 230-234

[77] Cantisani V, Maceroni P, D'Andrea V, Patrizi G, Di Segni M, De Vito C, Grazhdani $\mathrm{H}$ et al. Strain ratio ultrasound elastography increases the accuracy of colour-Doppler ultrasound in the evaluation of Thy-3 nodules. A bi-centre university experience. Eur Radiol 2016; 26 : 1441-1449

[78] Cantisani V, Lodise P, Di Rocco G et al. Diagnostic accuracy and interobserver agreement of Quasistatic Ultrasound Elastography in the diagnosis of thyroid nodules. Ultraschall Med 2015; 36: 162-167. doi: $10.1055 / \mathrm{s}-0034-1366467$

[79] Xue J, Cao XL, Shi L, Lin CH, Wang J, Wang L. The diagnostic value of combination of TI-RADS and ultrasound elastography in the differentiation of benign and malignant thyroid nodules. Clin Imaging 2016; 40: 913-916

[80] Barr RG, Cosgrove D, Brock M, Cantisani V, Correas JM, Postema AW, Salomon G et al. WFUMB Guidelines and recommendations on the clinical use of ultrasound elastography: Part 5. Prostate. Ultrasound Med Biol 2017; 43: 27-48

[81] Kamoi K, Okihara K, Ochiai A, Ukimura O, Mizutani Y, Kawauchi A, Miki T. The utility of transrectal real-time elastography in the diagnosis of prostate cancer. Ultrasound Med Biol 2008; 34: 1025-1032

[82] Xu G, Feng L, Yao M, Wu J, Guo L, Yao X, Zhao L et al. A new 5-grading score in the diagnosis of prostate cancer with real-time elastography. Int J Clin Exp Pathol 2014; 7: 4128-4135

[83] Fujimoto K, Kato M, Kudo M, Yada N, Shiina T, Ueshima K, Yamada Y et al. Novel image analysis method using ultrasound elastography for noninvasive evaluation of hepatic fibrosis in patients with chronic hepatitis C. Oncology 2013; 84: (Suppl 1): 3-12

[84] Tatsumi C, Kudo M, Ueshima K, Kitai S, Ishikawa E, Yada N, Hagiwara S et al. Non-invasive evaluation of hepatic fibrosis for type $C$ chronic hepatitis. Intervirology 2010; 53: 76-81

[85] Friedrich-Rust M, Wunder K, Kriener S, Sotoudeh F, Richter S, Bojunga ], Herrmann $\mathrm{E}$ et al. Liver fibrosis in viral hepatitis: noninvasive assessment with acoustic radiation force impulse imaging versus transient elastography. Radiology 2009; 252: 595-604 